\title{
123|-Meta-lodobenzylguanidine Single-Photon Emission Computerized Tomography/Computerized Tomography Scintigraphy in the Management of Neuroblastoma
}

\begin{abstract}
Neuroblastoma is the most common pediatric extracranial solid tumor. High-risk neuroblastoma is the most frequent presentation with an overall survival of approximately $50 \%$. ${ }^{123}$ I-meta-iodobenzylguanidine $\left.{ }^{123} \mathrm{I}-\mathrm{mIBG}\right)$ scintigraphy in the assessment of the primary tumor and its metastases at diagnosis and after chemotherapy is a cornerstone imaging modality. In particular, the bulk of skeletal metastatic disease evaluated with ${ }^{123} \mathrm{I}-\mathrm{mIBG}$ at diagnosis and the following chemotherapy has a prognostic value. Currently, single-photon emission computerized tomography/ computerised tomography (SPECT/CT) is considered a fundamental part of ${ }^{123} \mathrm{I}-\mathrm{mIBG}$ scintigraphy. ${ }^{123}$ I-mIBG SPECT/CT is a highly specific and sensitive imaging biomarker and it has been the basis of all existing neuroblastoma trials requiring molecular imaging. The introduction of SPECT/CT has shown not only the heterogeneity of the mIBG uptake within the primary tumor but also the presence of completely mIBG nonavid metastatic lesions with mIBG-avid primary neuroblastomas. It is currently possible to semi-quantitatively assess tracer uptake with standardized uptake value, which allows a more precise evaluation of the tracer avidity and can help monitor chemotherapy response. The patchy mIBG uptake has consequences from a theranostic perspective and may partly explain the failure of some neuroblastomas to respond to ${ }^{131} \mathrm{I}-\mathrm{mIBG}$ molecular radiotherapy. Various positron emission tomography tracers, targeting different aspects of neuroblastoma cell biology, are being tested as possible alternatives to ${ }^{123} \mathrm{I}-\mathrm{mIBG}$.
\end{abstract}

Keywords: ${ }^{123}$ I-meta-iodobenzylguanidine scintigraphy, ${ }^{123}$ I-meta-iodobenzylguanidine single-photon emission computerized tomography/computerised tomography, high-risk neuroblastoma,

standardised uptake value

\section{Introduction}

Hybrid imaging devices with the ability to perform single-photon emission computerized tomography (SPECT) and computerized tomography (CT) have been clinically available for approximately two decades. The great expansion of positron emission tomography (PET) with CT has not resulted in diminished clinical use of SPECT/CT. Although PET/CT scanners have a higher sensitivity and a better spatial resolution, the SPECT/CT system presents some remarkable advantages: it is widely available due to a lower cost; it is a well-established technology and therefore easy to use; most radioisotopes decay with lower energy emission, thus giving a lower radiation dose; and their half-life is often longer than most of the PET tracers, making them better suited to image biological processes. $^{[1]}$ Also, with the advent of the newest 16 slice SPECT/CT devices, the CT component of a SPECT/CT study can be programmed in different ways according to the clinical need: from a very low dose CT for attenuation correction only, to a low dose CT for attenuation correction and image fusion, to contrast-enhanced fully diagnostic CT scan acquired immediately at the end of the SPECT study, with the patient in the same position.

A semi-quantitative assessment of tracer uptake is now possible with the standardised uptake value (SUV) being introduced in routine SPECT/scanning: This measurement, with its well-known limitations from its use in PET scanning, allows a more precise evaluation of the
This is an open access journal, and articles are distributed under the terms of the Creative Commons Attribution-NonCommercial-ShareAlike 4.0 License, which allows others to remix, tweak, and build upon the work non-commercially, as long as appropriate credit is given and the new creations are licensed under the identical terms.

For reprints contact: WKHLRPMedknow_reprints@wolterskluwer.com

\section{Lorenzo Biassoni, Laura Privitera ${ }^{1}$}

Department of Radiology, Great Ormond Street Hospital for Children, 'Department of Developmental Biology and Cancer Research, UCL GOS Institute of Child Health, London, UK
Address for correspondence: Dr. Lorenzo Biassoni, Department of Radiology, Nuclear Medicine Unit, Great Ormond Street Hospital for Children, Great Ormond Street, London, WC1N 3JH, UK.

E-mail:lbiassoni001@gmail. com;LLrenzo.Biassoni@gosh. nhs.uk

Received: 27-01-2020 Accepted: 28-01-2021 Published: 23-09-2021

Access this article online Website: www.ijnm.in

DOI: 10.4103/ijnm.ijnm_10_21

Quick Response Code:

How to cite this article: Biassoni L, Privitera L. ${ }^{123}$ I-meta-iodobenzylguanidine single-photon emission computerized tomography/computerized tomography scintigraphy in the management of neuroblastoma. Indian J Nucl Med 2021;36:293-9. 
tracer avidity in a particular area, which can help monitor the effectiveness of therapy. ${ }^{[2]}$

\section{Meta-iodobenzylguanidine in Neuroblastoma Staging}

Neuroblastoma, an embryonal tumor of the autonomic nervous system, is the most common pediatric extracranial solid tumour. It is characterized by a diverse natural history, ranging from rapid malignant progression to spontaneous regression. ${ }^{[3]}$ Several clinical and biological markers have been validated for risk stratification, including age, stage, histopathology, chromosomal aberrations, amplification of the MYCN oncogene, and response to induction chemotherapy. Patients are usually treated following a risk-adapted therapeutic strategy. ${ }^{[4]}$

Contrast-enhanced $\mathrm{CT}$ and magnetic resonance imaging (MRI) are essential cross-sectional imaging modalities to characterize the primary tumor and demonstrate its relationship with the surrounding anatomical structures. ${ }^{[5]} \mathrm{CT}$ or MRI examinations have been very helpful in identifying imaging defined risk factors (IDRFs), such as, for example, encasement of blood vessels or brachial plexus nerves, compression of the trachea or major bronchi, infiltration of nearby organs or anatomical spaces (for example, the spinal cord or the peri-portal spaces). Based on the presence or absence of IDRFs, the primary neuroblastoma can be classified as localised (L1) or locally advanced (L2) and therefore, surgically resectable or unresectable. ${ }^{[6]}$

${ }^{123}$ I-meta-iodobenzylguanidine (mIBG) is a norepinephrine (NE) analogue with specific uptake in tumors derived from tissues of sympathetic nervous system origin, which express the norepinephrine transporter (NET) ${ }^{[7]}$ It was introduced in clinical practice in the mid-1980s. Initially labeled with ${ }^{131} \mathrm{I}$, labeling with ${ }^{123} \mathrm{I}$ was soon introduced, with significant advantages in terms of image quality and reduced radiation burden. Unfortunately, ${ }^{123} \mathrm{I}$ is cyclotron produced and not available in several countries. Approximately $90 \%$ of neuroblastomas express the NET and therefore are mIBG-avid, with approximately $10 \%$ being mIBG nonavid. ${ }^{[8]}$

Assessment of the primary tumor and its metastases with ${ }^{123} \mathrm{I}-\mathrm{meta}-\mathrm{iodobenzylguanidine} \quad\left({ }^{123} \mathrm{I}-\mathrm{mIBG}\right)$ scintigraphy at diagnosis and after induction chemotherapy is essential to stage the disease. ${ }^{[5,9]}$ Patients with distant metastatic disease (stage $\mathrm{M}$ ) fall in the high-risk category and are treated with a multi-drug, often dose-intensive, chemotherapy regimen. ${ }^{[4]}$

Clinically validated scoring methods to quantify the bulk of skeletal metastatic disease have been introduced. ${ }^{[10-12]}$ As neuroblastoma tends to metastasise predominantly within the bone marrow and cortical bone, assessing the bulk of skeletal metastatic disease with ${ }^{123}$ I-mIBG at diagnosis and its response following chemotherapy has been shown to have a prognostic value. A significant reduction of mIBG-avid metastatic disease after induction chemotherapy carries a better prognosis with both the European and the American scoring methods. ${ }^{[10,13]}$

In the management of neuroblastoma patients ${ }^{123} \mathrm{I}-\mathrm{mIBG}$ scintigraphy is used in the following clinical contexts: at diagnosis to assess the tumor avidity for the tracer and to stage the disease; after induction chemotherapy to assess the response of the primary tumor and metastatic lesions; in the case of suspected recurrence; and before molecular radiotherapy with ${ }^{131} \mathrm{I}-\mathrm{mIBG}$, to assess the number and the avidity of the lesions. ${ }^{[14]}$

SPECT/CT is now considered an integral part of ${ }^{123} \mathrm{I}-\mathrm{mIBG}$ scintigraphy: it minimises the number of equivocal lesions, thus making imaging acquisition at 4 and $48 \mathrm{~h}$ redundant; ${ }^{[14-16]}$ it significantly improves the accuracy of anatomical localisation of the lesions, facilitating correlation of SPECT with possible equivocal lesions on recent MRI or contrast-enhanced diagnostic quality CT; it identifies possible mIBG nonavid components of the primary tumor and nonavid metastatic lesions demonstrated on MRI; it allows monitoring of the avidity of the primary neuroblastoma and metastatic lesions with SUV. ${ }^{[14]}$

\section{${ }^{123}$ I-meta-Iodobenzylguanidine Single-Photon Emission Computerized Tomography/ Computerized Tomography: Patient's Preparation and Acquisition Technique}

Thyroid blockade prior to tracer injection with either potassium iodate, Lugol iodine solution or potassium perchlorate is mandatory. ${ }^{[14,17]}$ Most of the drugs known to interfere with mIBG uptake are seldom used in children. However, some children with neuroblastoma can be hypertensive (for example, due to renal artery stenosis caused by extrinsic compression from the primary tumour), on some anti-hypertensive drugs with possible interference with mIBG uptake. Commonly used medications for asthma and cough containing sympathomimetics can also interfere with mIBG uptake. Poor tracer uptake in the salivary glands and in the heart are typical features suggesting pharmacological interference. An up-to-date list of drugs interfering with mIBG uptake is presented in the recently published EANM guidelines on molecular imaging in neuroblastoma. ${ }^{[14]}$

The administered activity is proportional to the patient's weight and ranges between 37 and $400 \mathrm{MBq} \cdot{ }^{[14]}$ Images are acquired $20-24 \mathrm{~h}$ after ${ }^{123} \mathrm{I}-\mathrm{mIBG}$ injection. Images acquired at $4-6 \mathrm{~h}$ and $48 \mathrm{~h}$ are no longer necessary. The collimator's choice is left to the nuclear medicine department. The use of a medium-energy collimator has been recommended both for planar and SPECT ${ }^{123}$ I images as more favorable to reduce septal penetration with sodium-iodide detector gamma cameras; ${ }^{[18]}$ however, some low energy general purpose collimators can be perfectly adequate. Images can be 
acquired as a whole-body scan in the anterior and posterior projections $(5 \mathrm{~cm} / \mathrm{min}$ scan speed) or spot views of the different body segments (max $10 \mathrm{~min}$ for each spot view). ${ }^{[14]}$ It is strongly advised to acquire skull views in the anterior/ posterior and right lateral/left lateral projections, to better visualise the base of the skull, which not infrequently can harbour metastatic lesions. In a child with a full bladder, a delayed static view of the pelvis should be attempted once the child has voided. Otherwise, the insertion of a bladder catheter may be considered, especially in a child with presacral primary neuroblastoma or metastatic lesions in the pelvis.

A SPECT acquisition protocol typically consists of 120 projections, in steps of 3 degrees each, in either continuous or step-and-shoot mode, 25-35 s/step, with a $128 \times 128$ matrix. The field of view of the SPECT is confirmed after the static planar images, and it is usually centred on the site of the primary tumor (torso or the abdomen and pelvis).

The field of view of the CT scan should be set after completion of the SPECT and does not need to include the width of the SPECT acquisition. Several protocols for low dose and ultra-low-dose CT acquisitions are available, and the parameters may vary among different SPECT/ CT scanners. A possible low-dose CT acquisition may include a voltage around $80-100 \mathrm{kVp}$ and a tube current of approximately $10-40 \mathrm{mAs}$, with a given radiation dose within a range of $0.2-0.5 \mathrm{mSv}^{[19]}$

The ${ }^{123}$ I-mIBG scan with SPECT/CT is a lengthy study lasting at least $90 \mathrm{~min}$, and the highest image quality is paramount. As neuroblastoma is much more frequent in a patients' population aged between 1 and 5 years, compliance with the study, especially avoiding motion artefacts, can be difficult. Therefore, the child is likely to need some form of sedation or even general anesthesia if pediatric anaesthetic support is available in the hospital.

The main pitfall of a SPECT/CT acquisition during a ${ }^{123} \mathrm{I}-\mathrm{mIBG}$ scan is related to motion artefacts. In the case of significant patient's movement during the SPECT study or between the SPECT and the CT, correction of possible misregistration between the unenhanced CT and the SPECT can be attempted with one of the commercially available software programs using as landmarks the bladder, the liver (where there is a normal distribution of ${ }^{123} \mathrm{I}-\mathrm{mIBG}$ ), the heart (if included in the SPECT field of view).

\section{${ }^{123}$ I-meta-Iodobenzylguanidine Single-photon Emission Computerised Tomography/ Computerized Tomography Scan at Diagnosis}

The ${ }^{123} \mathrm{I}-\mathrm{mIBG}$ scintigraphy at diagnosis is essential to assess the mIBG avidity of the primary tumor $(8 \%-10 \%$ of neuroblastomas are mIBG nonavid) and establish the possible presence of distant metastases. The most frequent metastatic sites for neuroblastoma are the skeleton (bone marrow and cortical bone), followed by regional and distant lymph nodes, and the liver. Brain metastases are infrequent but possible. ${ }^{[20]}$ Lung metastases are very rare. Distant metastatic disease in a child older than 18 months of age at diagnosis represents a high-risk factor, requiring multi-drug dose-intensive induction chemotherapy.

The bulk of mIBG-avid skeletal metastatic disease at diagnosis estimated with validated scoring systems (SIOPEN and Curie) has prognostic value: a SIOPEN score $<3$ and a Curie score $<2$ carry a better prognosis than a score $>3$ and 2 , respectively. ${ }^{[13,21]} \mathrm{mIBG}$ nonavid primary neuroblastoma has been shown to have better survival than mIBG avid disease. ${ }^{[8]}$ Strong ${ }^{123}$ I-mIBG uptake in primary neuroblastic tumors, especially when associated with high serum levels of neuron-specific enolase, can be predictive of unfavourable histopathology and shorter event-free survival. ${ }^{[8,22]} \mathrm{mIBG}$ avidity of the primary tumor does not correlate with restricted diffusion at diffusion-weighted MRI (DW-MRI). ${ }^{[23]}$

Both the MRI scan of the primary neuroblastoma at diagnosis and the ${ }^{123} \mathrm{I}-\mathrm{mIBG}$ scintigraphy with SPECT/ CT shows the cellular heterogeneity of the primary tumor [Figure 1]. The introduction of SPECT/CT has significantly improved the detection of mIBG-avid lesions $^{[14]}$ clearly demonstrating lesions not visible or faintly demonstrated on planar images [Figure 2].

Primary tumor masses are often made of viable tumor nodules returning different signals on MRI, associated with foci of necrosis, hemorrhage, and calcification. ${ }^{[24]}$ In this context ${ }^{123}$ I-mIBG SPECT/CT often shows heterogeneous mIBG avidity within the primary tumor mass: nodules of viable tumor on MRI can exhibit a high, moderate, low or absent mIBG avidity on SPECT/CT. ${ }^{[23]}$ This is relevant when molecular radiotherapy with ${ }^{131} \mathrm{I}-\mathrm{mIBG}$ is contemplated: given the different degree of $\mathrm{mIBG}$ avidity (or no avidity at all), the uptake of the therapeutic radiopharmaceutical within the neuroblastoma lesions will be higher or lower, or completely absent, with more or less or no response. ${ }^{[25]}$ Initial attempts at testing the avidity of the primary neuroblastoma to different imaging biomarkers (in particular, ${ }^{123} \mathrm{I}-\mathrm{mIBG}$ targeting $\mathrm{NE}$ analog re-uptake, and ${ }^{68} \mathrm{Ga}$-DOTATATE targeting somatostatin receptors) have shown that some neuroblastoma cells show avidity for one tracer and not for the other, and vice versa: this finding needs further evaluation as it may have implications for molecular radiotherapy in that two combined therapeutic radiopharmaceuticals (for example, ${ }^{131} \mathrm{I}$-mIBG and ${ }^{177} \mathrm{~L} \mathrm{u}$-DOTATATE) may potentially yield a better response than one. ${ }^{[26]}$

\section{${ }^{123}$ I-meta-Iodobenzylguanidine Scan To Assess Response To Induction Chemotherapy}

${ }^{123} \mathrm{I}-\mathrm{mIBG}$ scintigraphy is very valuable for assessing the primary neuroblastoma response and the regional or distant metastases to induction chemotherapy. A great advantage of mIBG is its lack of uptake in reactive hyperplastic bone 

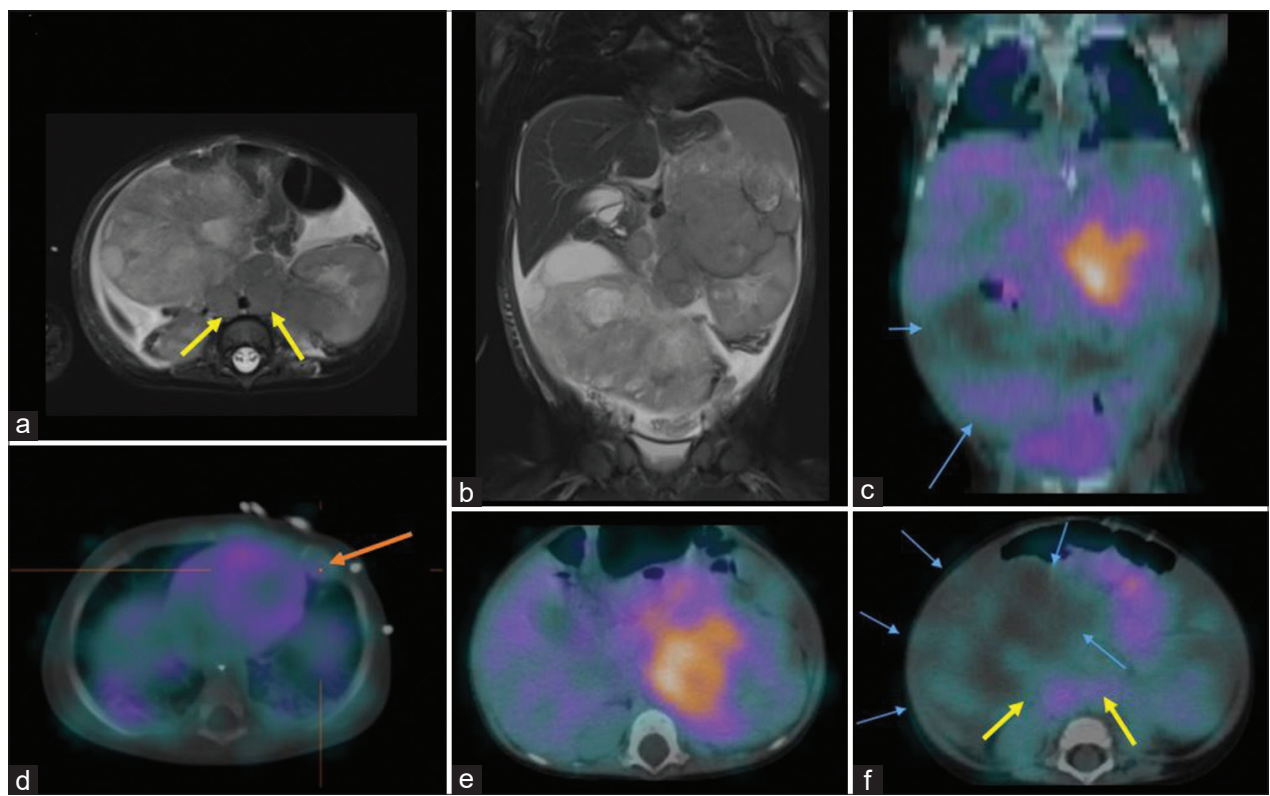

Figure 1: (a-f) 8-month-old girl with large neuroblastoma in the abdomen and pelvis at diagnosis. (c-f) ${ }^{123}$-meta-iodobenzylguanidine scintigraphy with single-photon emission computerized tomography/computerized tomography clearly shows the heterogeneous meta-iodobenzylguanidine uptake of the primary tumor (blue arrows). (d) Expansile meta-iodobenzylguanidine nonavid lytic metastatic lesion in a left rib with a mildly avid small soft-tissue nodule adjacent to it (orange arrow). (a and f) Enlarged left para-aortic and retrocaval meta-iodobenzylguanidine-avid lymph nodes (yellow arrows)

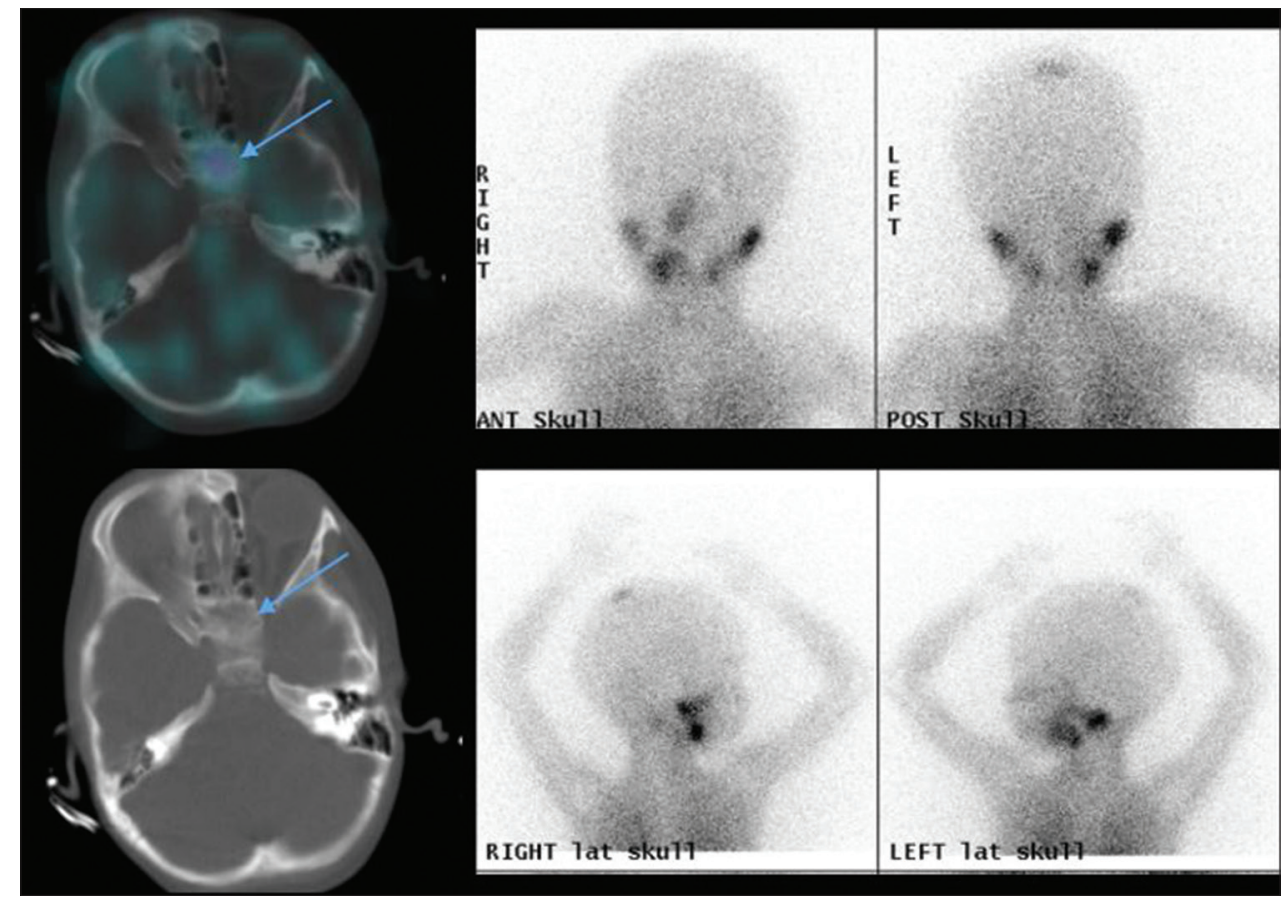

Figure 2: ${ }^{123}$-meta-iodobenzylguanidine scintigraphy with single-photon emission computerized tomography/computerized tomography in a 4-year-old girl with relapsed neuroblastoma. The image shows a metastatic lesion at the base of skull, not identified on planar images, clearly visible with single-photon emission computerized tomography/computerized tomography sequence

marrow, detecting residual or recurrent metastatic lesions easier. $^{[27]}$

The bulk of residual mIBG avid skeletal metastatic disease has been shown to be a prognostic indicator: a SIOPEN score $<3$ in the skeleton (curie score $<2$ ) following induction chemotherapy is associated with a better prognosis..$^{[13,21]}$
Persistent ${ }^{123} \mathrm{I}-\mathrm{mIBG}$ uptake with unchanged intensity in primary neuroblastoma after induction chemotherapy is likely to be associated with a poorer survival rate compared to postchemotherapy primary neuroblastomas, of a visible size on MRI, with no residual ${ }^{123}$ I-mIBG uptake (Biassoni L, unpublished data). Residual ${ }^{123}$ I-mIBG avidity in the primary tumor does not 
correlate with restricted diffusion at DWI-MRI after chemotherapy. ${ }^{[23]}$

Preoperative ${ }^{123} \mathrm{I}-\mathrm{mIBG} \quad \mathrm{SPECT} / \mathrm{CT} \quad$ scintigraphy after induction chemotherapy can be performed with contrast-enhanced diagnostic quality CT, especially when using the latest 16 slices SPECT/CT scanners: this protocol is highly efficient as it combines two diagnostic quality scans in one sitting, often with one sedation/ general anaesthesia, but should be done only if it avoids another diagnostic quality CT on a different session. ${ }^{[28]}$ Administration of i.v. radiological contrast does not create a problem to correct for photon attenuation from the SPECT if the topogram for attenuation correction is done before the radiotracer injection. The appropriate acquisition parameters for a diagnostic quality CT can be utilised on the SPECT/ CT scanner. Radio-opaque radiological contrast (omnipaque 300 ) at a dose of $2 \mathrm{ml} / \mathrm{kg}$ (maximum $75 \mathrm{ml}$ ) and at a flow rate of $1.8-2.5 \mathrm{ml} / \mathrm{s}$ is injected intravenously. The CT is programmed with a current intensity varying between $80 \mathrm{mAs}$ (chest) and 120-130 mAs (abdomen) and a voltage of $110 \mathrm{kVp}$, with a pitch of 1.5 and a slice thickness of $3 \mathrm{~mm}$. The CT is usually acquired with contrast injected in porto-venous phase, to allow proper visualisation of both the arteries and veins in the primary neuroblastoma region, thus allowing surgical planning. The blood vessels in the region of the primary tumor have to be clearly visible and their relationship with the primary tumor accurately described in the report [Figure 3].

The SUV estimated on the SPECT/CT dataset can be valuable to monitor changes in the $\mathrm{mIBG}$ avidity of the primary tumor after chemotherapy ${ }^{[29]}$ [Figure 4].

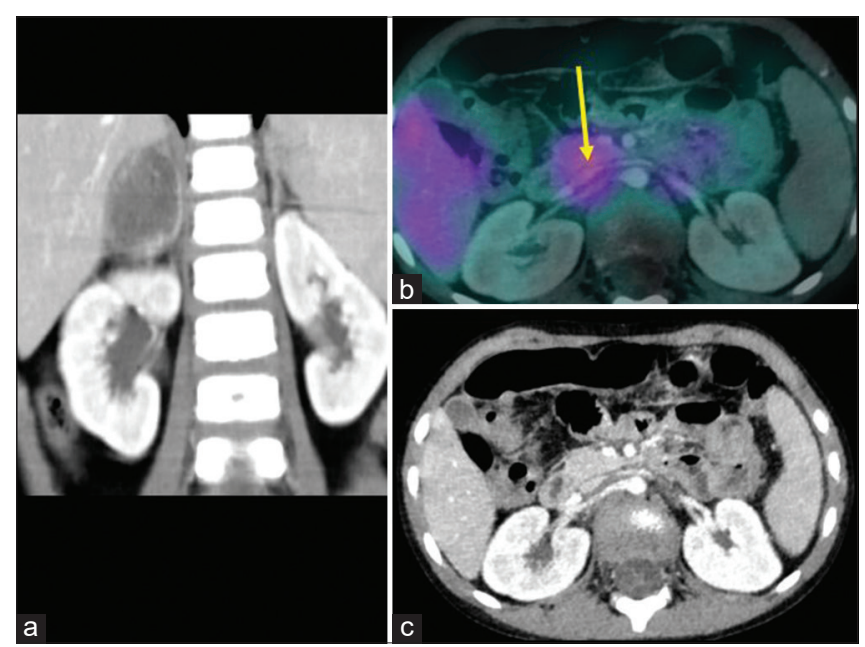

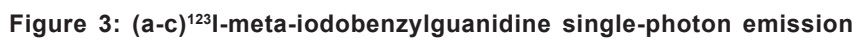
computerized tomography with contrast-enhanced diagnostic quality computerized tomography after induction chemotherapy of a 4-year-old boy with stage $M$ neuroblastoma. The meta-iodobenzylguanidine-avid primary tumor encases the right renal vessels (yellow arrow, b). Because of this imaging defined risk factor surgery was postponed and further chemotherapy was administered.
Strengths and Limitations
${ }^{123}$ i-Meta-Iodobenzylguanidine Scintigraphy

of

The main strength of the ${ }^{123} \mathrm{I}-\mathrm{mIBG}$ scan is its specificity. There is no tracer uptake in reactive or inflammatory tissue; in particular, no background uptake occurs in the normal skeleton, where the vast majority of metastatic lesions from neuroblastoma are located, making detection of metastases much easier. ${ }^{123} \mathrm{I}-\mathrm{mIBG}$ has been extensively and thoroughly tested and validated, and all the clinical trials in neuroblastoma requiring molecular imaging to assess the results are currently based on ${ }^{123} \mathrm{I}$-mIBG.

A significant limitation of the ${ }^{123} \mathrm{I}-\mathrm{mIBG}$ scintigraphy with SPECT/CT is its complexity. Sedation/general anesthesia are often necessary to keep the child still and acquire high-quality images. The need to scan at $24 \mathrm{~h}$ after tracer injection is inconvenient for the child and family, who have to come to the hospital on 2 subsequent days. The radiation dose from the ${ }^{123} \mathrm{I}-\mathrm{mIBG}$ component of the study depends on the injected activity and the child's age, and is usually moderately high $(4-7 \mathrm{mSv}$, depending on the child's age - the younger the child the higher the dose); the low dose CT only adds a negligible dose $(0.2-0.4 \mathrm{mSv})$ if precautions are taken to not irradiate unnecessary parts of the body and if low-dose CT protocols are used. Another drawback of ${ }^{123} \mathrm{I}-\mathrm{mIBG}$ comes from its being a gamma camera SPECT tracer, with the related limited sensitivity and spatial resolution in comparison to a PET tracer. Furthermore, the need to administer a thyroid blocking agent adds to the complexity of the examination. In addition, ${ }^{123} \mathrm{I}-\mathrm{mIBG}$ is not available in several countries, obliging them to use ${ }^{131} \mathrm{I}-\mathrm{mIBG}$ scan for diagnostic purposes, which is highly unsatisfactory because of the poor quality images with a high radiation burden. ${ }^{18} \mathrm{~F}-\mathrm{FDG}$ PET is a better alternative. ${ }^{[30]}$

\section{Alternative Tracers in Neuroblastoma}

Several PET tracers are being tested as possible alternatives to ${ }^{123} \mathrm{I}-\mathrm{mIBG}$ or in $\mathrm{mIBG}$ negative neuroblastoma. The recent EANM guidelines on molecular imaging in neuroblastoma present an overview of the advantages and disadvantages of the currently available PET tracers. ${ }^{[14]}$

\section{Conclusion}

Neuroblastoma is a childhood malignancy with heterogeneous histopathology and a highly varied natural history. High-risk neuroblastoma is the most frequent presentation and the overall survival is still only around $40 \%-50 \%,{ }^{[31]}$ with a significant risk of serious side effects and second malignancies due to treatment-related toxicity. ${ }^{[32]}{ }^{123}$ I-mIBG SPECT/CT is a highly specific and sensitive thoroughly tested imaging biomarker and has been the cornerstone of all existing neuroblastoma trials requiring molecular imaging. The addition of SPECT/ $\mathrm{CT}$ has clearly shown the heterogeneity of the primary 


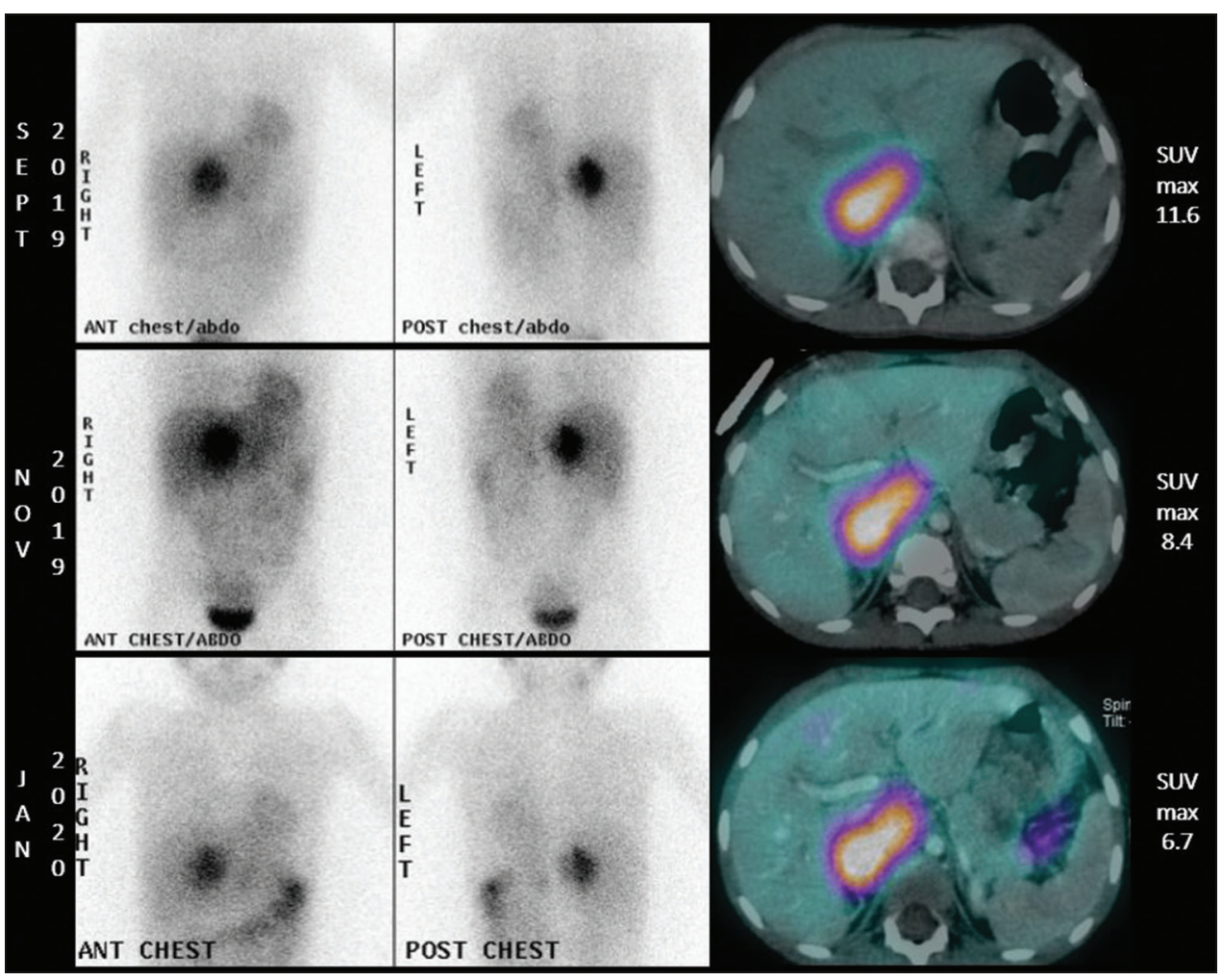

Figure 4: A 3-year-old boy with relapsed refractory neuroblastoma at different time points during follow-up. While no obvious change is visually identifiable on ${ }^{123}$-meta-iodobenzylguanidine scintigraphy planar images, the evaluation of the standardized uptake value on the single-photon emission computerized tomography/computerized tomography clearly shows a decreasing ${ }^{123}$-meta-iodobenzylguanidine avidity of the primary tumor during treatment

tumour, with areas of very different mIBG avidity within it; besides, the presence of completely mIBG nonavid metastatic lesions in mIBG avid primary neuroblastomas has become apparent. This heterogeneous mIBG avidity of neuroblastoma has consequences from a theranostic perspective and could possibly explain, at least in part, the overall disappointing results of ${ }^{131} \mathrm{I}-\mathrm{mIBG}$ molecular radiotherapy in some neuroblastoma patients so far.

New PET tracers targeting different aspects of neuroblastoma cell biology, which could allow a theranostic approach with high energy radiopharmaceuticals for effective molecular radiotherapy, even possibly in combination, are needed.

\section{Financial support and sponsorship}

Nil.

\section{Conflicts of interest}

There are no conflicts of interest.

\section{References}

1. Mariani G, Bruselli L, Kuwert T, Kim EE, Flotats A, Israel O, et al. A review on the clinical uses of SPECT/CT. Eur J Nucl Med Mol Imaging 2010;37:1959-85.

2. Bailey DL, Willowson KP. Quantitative SPECT/CT: SPECT joins PET as a quantitative imaging modality. Eur J Nucl Med Mol Imaging 2014;41 Suppl 1:S17-25.

3. Maris JM. Recent advances in neuroblastoma. N Engl J Med
2010;362:2202-11.

4. Matthay KK, Maris MJ, Schleiermacher G, Nakagawara A, Mackall CL, Diller L, et al. Neuroblastoma. Nat Rev Dis Primers. 2016 Nov 10;2:16078. doi: 10.1038/nrdp.2016.78.

5. Brisse HJ, McCarville MB, Granata C, Krug KB, Wootton-Gorges SL, Kanegawa K, et al. Guidelines for imaging and staging of neuroblastic tumors: Consensus report from the International Neuroblastoma Risk Group Project. Radiology 2011;261:243-57.

6. Siegel MJ, Jaju A. MR imaging of neuroblastic masses. Magn Reson Imaging Clin N Am 2008;16:499-513, vi.

7. Dubois SG, Geier E, Batra V, Yee SW, Neuhaus J, Segal M, et al. Evaluation of norepinephrine transporter expression and metaiodobenzylguanidine avidity in neuroblastoma: A Report from the Children's Oncology Group. Int J Mol Imaging 2012;2012:250834.

8. DuBois SG, Mody R, Naranjo A, Van Ryn C, Russ D, Oldridge D, et al. MIBG avidity correlates with clinical features, tumor biology, and outcomes in neuroblastoma: A report from the Children's Oncology Group. Pediatr Blood Cancer 2017;64:e26545.

9. Park JR, Bagatell R, Cohn SL, Pearson AD, Villablanca JG, Berthold $\mathrm{F}$, et al. Revisions to the international neuroblastoma response criteria: A consensus statement from the national cancer institute clinical trials planning meeting. J Clin Oncol 2017;35:2580-7.

10. Yanik GA, Parisi MT, Shulkin BL, Naranjo A, Kreissman SG, London WB, et al. Semiquantitative $\mathrm{mIBG}$ scoring as a prognostic indicator in patients with stage 4 neuroblastoma: A Report from the Children's Oncology Group. J Nucl Med 2013;54:541-8.

11. Lewington $\mathrm{V}$, Lambert $\mathrm{B}$, Poetschger U, Sever ZB, 
Giammarile F, McEwan AJ, et al. 123I-mIBG scintigraphy in neuroblastoma: Development of a SIOPEN semiquantitative reporting method by an international panel. Eur J Nucl Med Mol Imaging 2017;44:234-41.

12. Decarolis B, Schneider C, Hero B, Simon T, Volland R, Roels F, et al. Iodine-123 metaiodobenzylguanidine scintigraphy scoring allows prediction of outcome in patients with stage 4 neuroblastoma: Results of the Cologne interscore comparison study. J Clin Oncol 2013;31:944-51.

13. Ladenstein $R$, Lambert $B$, Pötschger $U$, Castellani MR, Lewington V, Bar-Sever Z, et al. Validation of the mIBG skeletal SIOPEN scoring method in two independent high-risk neuroblastoma populations: The SIOPEN/HR-NBL1 and COG-A3973 trials. Eur J Nucl Med Mol Imaging 2018;45:292-305.

14. Bar-Sever Z, Biassoni L, Shulkin B, Kong G, Hofman MS, Lopci $\mathrm{E}$, et al. Guidelines on nuclear medicine imaging in neuroblastoma. Eur J Nucl Med Mol Imaging 2018;45:2009-24.

15. Liu B, Servaes S, Zhuang H. SPECT/CT MIBG imaging is crucial in the follow-up of the patients with high-risk neuroblastoma. Clin Nucl Med 2018;43:232-8.

16. Černý I, Prášek J, Kašpárková H. Superiority of SPECT/CT over planar 123I-mIBG images in neuroblastoma patients with impact on Curie and SIOPEN score values. Nuklearmedizin 2016;55:151-7.

17. Thurlow B, Morris E, Price L, Melhuish T, Michopoulou S, King S, et al. A comparison of thyroid blockade strategies in paediatric 123I-meta-iodobenzylguanidine scanning: A dual centre study. Nucl Med Commun 2020;41:436-42.

18. Snay ER, Treves ST, Fahey FH. Improved quality of pediatric 123I-MIBG images with medium-energy collimators. J Nucl Med Technol 2011;39:100-4.

19. Gelfand MJ, Lemen LC. PET/CT and SPECT/CT dosimetry in children: The challenge to the pediatric imager. Semin Nucl Med 2007;37:391-8.

20. Matthay KK, Edeline V, Lumbroso J, Tanguy LM, Asselain B, Zucker JM, et al. Correlation of early metastatic response by 123I-metaiodobenzylguanidine scintigraphy with overall response and event-free survival in stage IV neuroblastoma. J Clin Oncol Off J Am Soc Clin Oncol 2003;21:2486-91.

21. Yanik GA, Parisi MT, Naranjo A, Nadel H, Gelfand MJ, Park JR, et al. Validation of postinduction curie scores in high-risk neuroblastoma: A children's oncology group and siopen group report on siopen/HR-NBL1. J Nucl Med 2018;59:502-8.

22. Fendler WP, Wenter V, Thornton HI, Ilhan H, von Schweinitz D, Coppenrath E, et al. Combined scintigraphy and tumor marker analysis predicts unfavorable histopathology of neuroblastic tumors with high accuracy. PLoS One 2015;10:e132809.

23. Privitera L, Hales PW, Musleh L, Morris E, Sizer N, Barone G, et al. Comparison between diffusion-weighted MRI and 123I-mIBG uptake in primary high-risk neuroblastoma. J Magn Reson Imaging 2020. doi: 10.1002/jmri.27458.

24. Humphries PD, Sebire NJ, Siegel MJ, Olsen ØE. Tumors in pediatric patients at diffusion-weighted MR imaging: Apparent diffusion coefficient and tumor cellularity. Radiology 2007;245:848-54.

25. Agrawal A, Rangarajan V, Shah S, Puranik A, Purandare N. MIBG (metaiodobenzylguanidine) theranostics in pediatric and adult malignancies. Br J Radiol 2018;91:20180103.

26. Gains JE, Aldridge MD, Mattoli MV, Bomanji JB, Biassoni L, Shankar A, et al. 68Ga-DOTATATE and 123I-mIBG as imaging biomarkers of disease localisation in metastatic neuroblastoma: Implications for molecular radiotherapy. Nucl Med Commun 2020;41:1169-77.

27. Sharp SE, Trout AT, Weiss BD, Gelfand MJ. MIBG in Neuroblastoma Diagnostic Imaging and Therapy. Radiographics 2016;36:258-78.

28. Nadel HR. SPECT/CT in pediatric patient management. Eur J Nucl Med Mol Imaging 2014;41 Suppl 1:S104-14.

29. Brady SL, Shulkin BL. Analysis of quantitative [I-123] mIBG SPECT/CT in a phantom and in patients with neuroblastoma. : EJNMMI Phys. 2019;6:31. doi: 10.1186/s40658-019-0267-6.

30. Dhull VS, Sharma P, Patel C, Kundu P, Agarwala S, Bakhshi S, et al. Diagnostic value of $18 \mathrm{~F}-\mathrm{FDG} \mathrm{PET} / \mathrm{CT}$ in paediatric neuroblastoma: Comparison with 131I-MIBG scintigraphy. Nucl Med Commun 2015;36:1007-13.

31. Pinto NR, Applebaum MA, Volchenboum SL, Matthay KK, London $\mathrm{WB}$, Ambros PF, et al. Advances in risk classification and treatment strategies for neuroblastoma. J Clin Oncol Off J Am Soc Clin Oncol 2015;33:3008-17.

32. Applebaum MA, Vaksman Z, Lee SM, Hungate EA, Henderson TO, London WB, et al. Neuroblastoma survivors are at increased risk for second malignancies: A report from the international neuroblastoma risk group project. Eur J Cancer 2017;72:177-85. 